\title{
छs \\ Fractal Dimension and Localization of DNA Knots
}

\author{
Erika Ercolini, ${ }^{1}$ Francesco Valle, ${ }^{1, *}$ Jozef Adamcik, ${ }^{1}$ Guillaume Witz, ${ }^{1}$ Ralf Metzler, ${ }^{2, \dagger}$ Paolo De Los Rios, ${ }^{3}$ \\ Joaquim Roca, ${ }^{4}$ and Giovanni Dietler ${ }^{1}$ \\ ${ }^{1}$ Laboratory of Physics of Living Matter, IPMC, Ecole Polytechnique Fédérale de Lausanne (EPFL), CH-1015 Lausanne, Switzerland \\ ${ }^{2}$ Nordic Institute for Theoretical Physics (NORDITA), Blegdamsvej 17, 2100 Kobenhavn Ø, Denmark \\ ${ }^{3}$ Laboratory of Statistical Biophysics, ITP, Ecole Polytechnique Fédérale de Lausanne (EPFL), CH-1015 Lausanne, Switzerland \\ ${ }^{4}$ Instituto de Biologia Molecular de Barcelona, CID-CSIC, Jordi Girona 18-26, 08034 Barcelona, Spain
}

(Received 13 July 2006; published 29 January 2007)

\begin{abstract}
The scaling properties of DNA knots of different complexities were studied by atomic force microscope. Following two different protocols DNA knots are adsorbed onto a mica surface in regimes of (i) strong binding, that induces a kinetic trapping of the three-dimensional (3D) configuration, and of (ii) weak binding, that permits (partial) relaxation on the surface. In (i) the radius of gyration of the adsorbed DNA knot scales with the 3D Flory exponent $\nu \approx 0.60$ within error. In (ii), we find $\nu \approx 0.66$, a value between the $3 \mathrm{D}$ and $2 \mathrm{D}(\nu=3 / 4)$ exponents. Evidence is also presented for the localization of knot crossings in $2 \mathrm{D}$ under weak adsorption conditions.
\end{abstract}

DOI: 10.1103/PhysRevLett.98.058102

The first systematic study of knots was undertaken by Tait in the 19th century [1], following Kelvin's theory of vortex atoms [2]. During the 20th century progress was made understanding knots in a topological framework and invariants were found to classify them [3,4]. Experimentally, knots remained elusive and difficult to study, but the discovery of their role in biological processes $[5,6]$ revived the interest in their properties. For example, knots on DNA inhibit its separation into single strands during replication, impede access to the full genetic code during transcription, are implicated in gene regulation [7], and influence DNA stability [8]. Replication and transcription of circular DNA are controlled by topoisomerases [5] promoting questions on the detailed mechanism of enzymatic knot detection [9]. Finally, knots have been found in proteins in their native states [10]. The physical interest in the behavior of DNA knots concerns two main questions: (i) the scaling properties of the radius of gyration $R_{g}$ [11] and (ii) knot localization.

(i) From simulations and scaling arguments, it is commonly accepted that the gyration radius of knots to leading order scales as $R_{g} \simeq A \mathcal{L}^{\nu}$, for all knot types, as long as the polymer is sufficiently long [12-14], where $\mathcal{L}$ is the contour length. Here, we quantify the Flory exponent $\nu$ of $3 \mathrm{D}$ and $2 \mathrm{D}$ configurations by determining the fractal dimension of single DNA knots.

(ii) From a polymer physics interest, and to understand better the action of topoisomerases and the physiological role of DNA knots, it is crucial to find out whether knots segregate into simply connected rings, with all essential crossings confined in a knot region of contour length $s$ much smaller than the overall chain length $\mathcal{L}$. Such localization has been predicted theoretically in 2D as a consequence of entropic maximization [15]. Simulations in 3D yield a size distribution of the knot region that is peaked well below $\mathcal{L}$ for fixed knot types [16], and the size $s$ of the
PACS numbers: 87.64.Dz, 36.20.Ey, 82.35.Gh, 87.14.Gg

knot region scales as $s \sim \mathcal{L}^{t}$, with $t<1$ [13,14]. It is experimentally difficult to probe the predicted scaling behavior $s \sim \mathcal{L}^{t}$, since $\mathcal{L}$ would have to be varied significantly. This is at present out of reach given the available techniques used to prepare the DNA knots.

Here we study the scaling properties and chain configuration of DNA knots adsorbed onto a mica surface by atomic force microscope (AFM). Under strong trapping conditions, we find that the gyration radius $R_{g}$ scales with the $3 \mathrm{D}$ exponent $\nu \approx 0.58$, while for weak trapping a larger value is observed, $\nu \approx 0.66$. Moreover, from the analysis of single chain configurations we conclude that simple knots localize, as predicted from previous studies.

Usually, knotted DNA obtained by topoisomerases is studied by electron microscopy (EM) [17,18]. For sufficient contrast at the crossings, EM imaging requires coating of DNA by the protein RecA, causing pronounced changes of physical parameters of DNA such as stiffness and apparent diameter $[5,6,18]$. As we document here, AFM can provide high resolution images of bare DNA, permitting to probe its unmasked polymeric properties. AFM has been applied to the visualization of catenanes, resolving the crossings without protein coating [19]. Being a surface technique probing the $2 \mathrm{D}$ properties of the adsorbed DNA, AFM does not give direct access to the 3D conformation. However, we have shown that such information can indeed be extracted from AFM images [20]. In particular, we obtain the scaling exponent $\nu$ by determining the (average) fractal dimension $d_{f}=1 / \nu$ of individual DNA knot configurations [21]. The configuration is found at single molecule level from AFM images of DNA knots adsorbed onto a flat surface out of solution and imaged by AFM in air. We consider two cases: (i) strong adsorption of DNA knots on 3-aminopropyltriethoxy silane (APTES) modified mica; this strong kinetic trapping roughly corresponds to a projection of the DNA knot onto the APTES- 
mica surface. As the fractal dimension of a noncompact polymer in $3 \mathrm{D}$ fulfills $d_{f} \leq 2$, its projection onto a $2 \mathrm{D}$ surface preserves the value of $d_{f}$ [21], and we can indeed infer the scaling exponent of the 3D structure. (ii) The second case is weak adsorption onto untreated mica in the presence of $\mathrm{Mg}^{2+}$ ions in solution. The ions act as bridges between the negative mica surface and DNA charges. In this case, the adsorption process allows for (partial) 2D relaxation of the knot configuration.

Knotted and unknotted DNA was isolated from P4 phage capsids according to the protocols given in [22,23]. All DNA knots are 10346 base pairs (bp) long, corresponding to a total contour length of $\approx 3.5 \mu \mathrm{m}$ for all knot types. The solutions contained a mixture of knot types with a minimal crossing number ranging up to $30-40$, with mean complexity close to 30 minimal crossings [23]. Further extraction by electroelution from agarose gels was performed to yield solutions containing DNA knots of low minimal crossing numbers in a range from 3 to $6[24,25]$. We cannot exclude that among these simple knots some could be unknots. The DNA knots were free from disturbing supercoiling since they were obtained by joining complementary ends without ligation of the strands (nicked DNA). Knotted DNA was diluted in a buffer solution of $10 \mathrm{mM}$ tris- $\mathrm{HCl}$, $p \mathrm{H} 7.6$ and stored at $4{ }^{\circ} \mathrm{C}$. For strong adsorption experiments [20], the freshly cleaved mica substrate was positively charged by exposing it to 3-aminopropyltriethoxy silane (APTES) vapors during $2 \mathrm{~h}$ at room temperature in a dry atmosphere [26]. A $10 \mu \mathrm{l}$ drop of a knotted DNA solution was deposited onto the substrate surface for $10 \mathrm{~min}$ and then rinsed with ultrapure water (USF, Elga). For the weak adsorption experiments, $10 \mu \mathrm{l}$ DNA knot solution (in TE buffer containing $5 \mathrm{mM} \mathrm{MgCl}$ ) was deposited on freshly cleaved mica for $10 \mathrm{~min}$ and then the sample was rinsed with ultrapure water (see above). The samples were finally blown dry with air. The DNA images were recorded by means of an AFM operated in intermittent-contact mode, in order to reduce the effect of lateral forces during scanning of the surface. For the case of strong adsorption, we checked that the sample remains stable for weeks if kept in dry atmosphere and that upon imaging in liquid the molecules do not rearrange, proving the irreversibility of the adsorption [20].

Figure 1 depicts typical images of four knotted DNA molecules obtained under strong (left column) and weak (right column) adsorption conditions. In the top row, knots with few essential crossings $(\leq 6)$ are shown, while the bottom row features more complex knots with up to 30 essential crossings. It is evident from these images that strong adsorption yields molecules with many crossings, although most of them are nonessential, while for the weak adsorption likely only the essential crossings are present. The latter case would indicate that the molecules are relaxed in a quasi-2D state.

The fractal dimension was determined from the images using the box counting algorithm calculating the number of

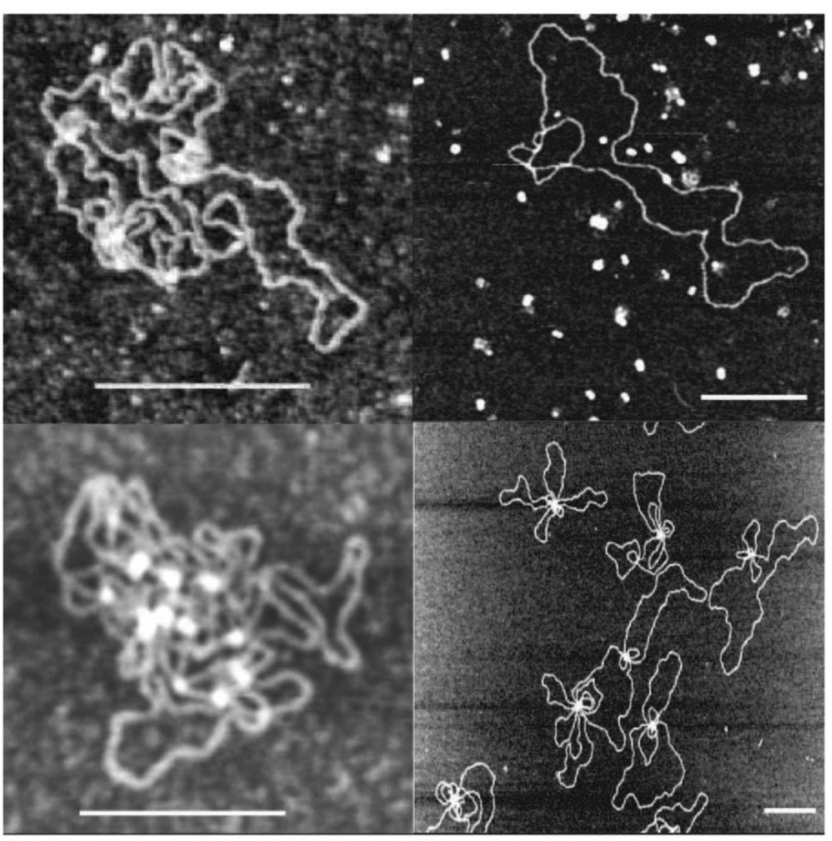

FIG. 1. AFM images of DNA knots selected from hundreds recorded. Strong adsorption case in the left column, weak adsorption on the right. Top row shows simple knots $(\leq 6$ essential crossings), bottom row complex knots (up to 30-40 essential crossings). Although statistically all knots behave similarly, single knot images clearly differ from each other. The scale bar represents $250 \mathrm{~nm}$ on all images.

boxes $N(L)$ containing a part of the molecule as a function of the box size $L$ [21]. The curves for $N(L)$ present two scaling regimes, as shown in Fig. 2, similarly to the linear DNA case [20]. On length scales smaller than the crossover length $\ell_{p}$, DNA appears like a rigid rod with $d_{1} \approx 1$, while on scales larger than $\ell_{p}$, DNA appears flexible, and the relevant scaling exponent can be observed. We therefore

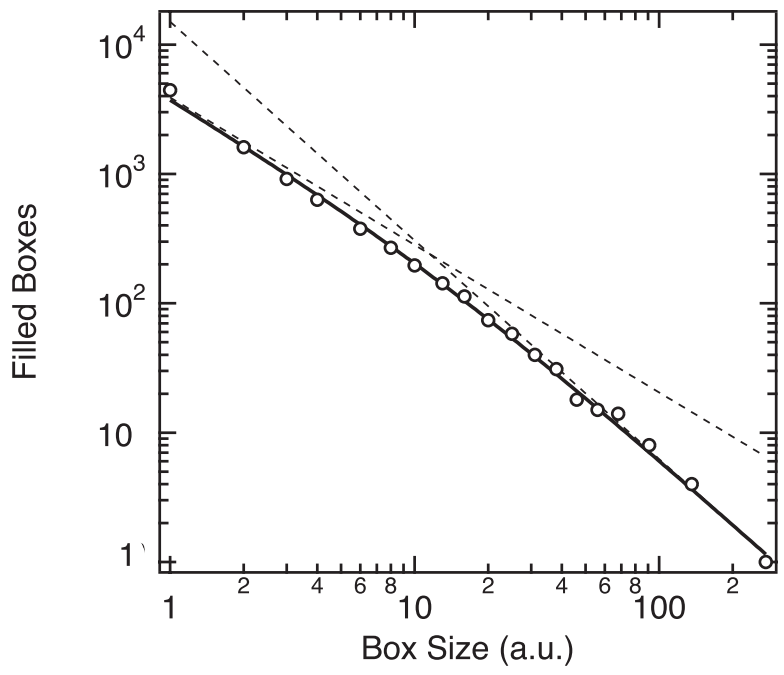

FIG. 2. Log-log plot of the number $N(L)$ of boxes of size $L$ filled with the knot versus $L$. The line is a fit to Eq. (1). 
interpret $\ell_{p}$ as the persistence length of DNA (for doublestranded DNA under normal conditions $\ell_{p} \approx 45 \mathrm{~nm}$ ).

To describe the crossover of $N(L)$ from the initial rigid rod to SAW behavior at $L=\ell_{p}$ we used the function

$$
N(L)=a\left(\frac{L}{\ell_{p}}\right)^{-d_{1}}\left(1+\frac{L}{\ell_{p}}\right)^{-\left(d_{f}-d_{1}\right)} .
$$

Each image of a DNA knot was put through the box counting algorithm and the resulting function was fitted with Eq. (1). The fractal dimension $d_{f}$ for both large and small length scales, and the persistence length $\ell_{p}$ from each individual molecule were then averaged to yield the overall quantities $\bar{d}_{f}$ and $\bar{\ell}_{p}$.

Table I summarizes the results for the fractal dimension and critical exponents for strongly adsorbed DNA knots, representing averages over 50 DNA knots. The errors are taken as the standard deviations for each case. Simple knots correspond to an essential crossing number $\leq 6$, while the complex knots comprise all knots in a wide distribution of essential crossings with the most probable number of essential crossings around 30 [23]. In Table I, the first line contains the values for the unknots, that were purified from the same gel as the knots by taking the slowest band. Similarly to the knots, this circular DNA is not supercoiled because it is nicked. The results provide the first experimental proof that circular and knotted DNA under strong adsorption conditions correspond to a geometrical projection of the $3 \mathrm{D}$ configuration, and that the fractal dimension $d_{f} \approx 1.7$ is preserved upon strong adsorption, consistent with the previous findings for linear DNA [20]. In particular, these results confirm the theoretical and numerical findings that the gyration radius of circular and knotted flexible polymers scales like $R_{g} \sim$ $\mathcal{L}^{\nu}$ with $\nu \approx 0.588[12-14,27]$.

Table II contains the results for the case when DNA knots were deposited in presence of $\mathrm{Mg}^{2+}$. The images in the right column of Fig. 1 clearly indicate that there are significantly fewer crossings than in the strong adsorption case (left column). One expects exponents that clearly differ from the $3 \mathrm{D}$ values, since relaxation should lead to conformations closer to the ones of 2D polymers, characterized by $\nu=0.75$. Yet, the scaling exponents for all three cases are significantly smaller, $\nu=0.66(1)<0.75$. This may indicate that only a partial $2 \mathrm{D}$ relaxation takes place under weak adsorption conditions, or, that we are in the

TABLE I. Fractal dimension $d_{f}$ and Flory exponent $\nu$ for the strong adsorption case.

\begin{tabular}{lcc}
\hline \hline & Strong adsorption & \\
\hline & $d_{f}$ & $\nu=1 / d_{f}$ \\
\hline Unknots & $1.711 \pm 0.042$ & $0.585 \pm 0.014$ \\
Simple knots & $1.685 \pm 0.055$ & $0.594 \pm 0.019$ \\
Complex knots & $1.835 \pm 0.076$ & $0.545 \pm 0.024$ \\
\hline \hline
\end{tabular}

presence of a different universality class [28]. These points need to be further clarified using longer DNA molecules, because the box counting algorithm can be affected by finite size effects and by the DNA thickness on AFM images.

In the analysis each knot was treated separately and data were fitted with Eq. (1). The results presented in Tables I and II are the averages of the fractal dimensions or of the corresponding critical exponents. This procedure avoids the problem of knowing exactly the knot type and to average over only one type of knot [27]. All values for $d_{1}$ were within $1.0 \pm 0.1$ corroborating the stiff rod behavior on lengths scales shorter than $\ell_{p}$.

The average persistence length $\ell_{p}$ gained from the fits with (1) was in the range 7-10 $\mathrm{nm}$ for strong adsorption (APTES-mica) and between 30-50 nm for weak adsorption $\left(\mathrm{Mg}^{2+}\right)$. The small values in the first case are probably due to the very many unessential crossings, making the apparent persistence length determined from Eq. (1) much shorter. In fact, if we analyze the traceable parts of the DNA molecules and apply the well-known relation $\left\langle\cos \left(\theta\left(L+L_{o}\right)-\theta\left(L_{o}\right)\right)\right\rangle=e^{-L / \ell_{p}}$, where $\theta(L)$ is the direction of the tangent to the curve at $L$, the values were in the range $30-50 \mathrm{~nm}$ for strong and weak adsorption conditions in agreement with the literature values [20,29-31].

Apart from the critical exponents and the persistence length, from the AFM images of weakly adsorbed simple knots we can deduce the localization behavior, as (almost) all nonessential crossings are removed during the slow trapping process. In Fig. 3 we present additional images of DNA knots deposited under weak adsorption conditions, illustrating the localization of the essential crossings within a small region of the chain when allowed to (partially) relax in 2D. The upper left image is an unknot of the same length as the knots and was extracted by electroelution from the first band of the agarose gels. Almost all unknots we have imaged had no crossings, such that the crossings on the other images, with significance, must be due to essential crossings. Such localization into a comparatively small knot region was predicted for 2D self-avoiding chains in Ref. [15]. This localization is significant, and therefore the polymer phase is different from dense or $\Theta$ conditions, for which delocalization was predicted [32].

While future studies with more advanced techniques to prepare longer knots are necessary to obtain more detailed information about the critical exponents (data over a few decades are needed to unambiguously determine a critical

TABLE II. Same as in Table I but for weak adsorption.

\begin{tabular}{lcc}
\hline \hline & Weak adsorption & \\
\hline & $d_{f}$ & $\nu=1 / d_{f}$ \\
\hline Unknots & $1.491 \pm 0.037$ & $0.670 \pm 0.017$ \\
Simple knots & $1.530 \pm 0.065$ & $0.654 \pm 0.028$ \\
Complex knots & $1.541 \pm 0.086$ & $0.650 \pm 0.036$ \\
\hline \hline
\end{tabular}




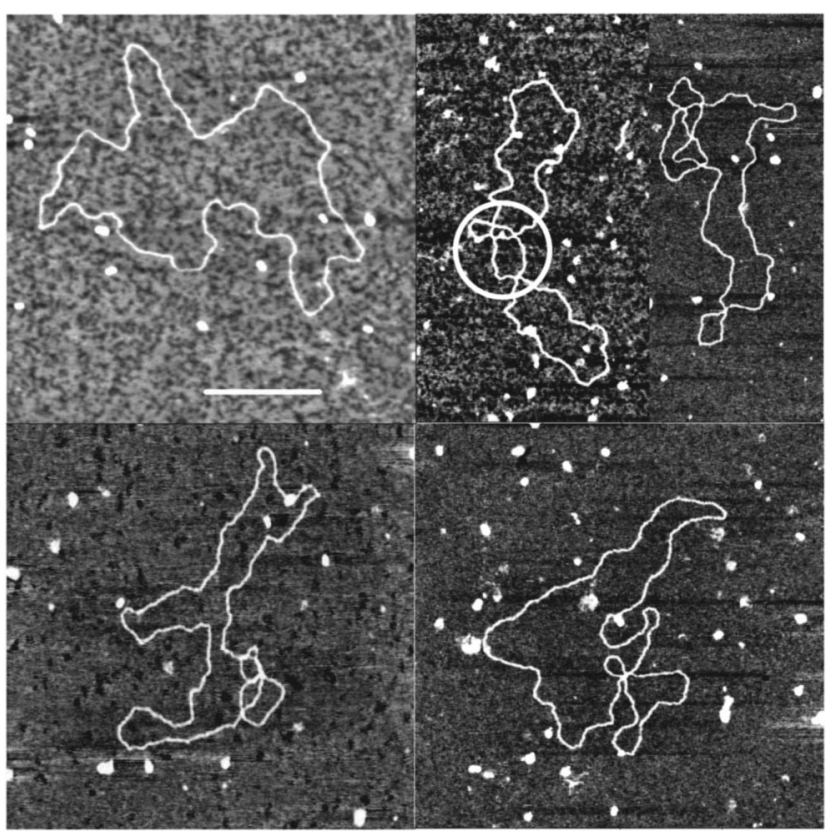

FIG. 3. Upper left: image of an unknot. The other images are DNA knots with a number of essential crossings smaller than 6 as separated from the agarose gels. Scale bar $250 \mathrm{~nm}$.

exponent [20]) and possible size effects as well as about the critical exponent $t$ governing localization, we demonstrated that AFM imaging and determination of the fractal dimension of the chain configuration provide an outstanding way to analyze the behavior of DNA knots.

We thank A. Stasiak, J. B. Schvartzman, C. Vanderzande for fruitful discussions and the Swiss National Science Foundation Grant No. 200021-101851, NSERC and the Canada Research Chairs program, and the Spanish Plan Nacional de I + D + I for support.

*Present address: Department of Biochemistry "G. Moruzzi," University of Bologna, Via Irnerio 48, 40126 Bologna, Italy

†Present address: Physics Department, University of Ottawa, ON, K1N 6N5, Canada.

[1] P. G. Tait, Trans. R. Soc. Edinburgh 28, 145 (1877).

[2] W. Thomson, Philos. Mag. 34, 15 (1867).

[3] L.H. Kauffman, Knots and Physics (World Scientific, Singapore, 1993).

[4] A.C.C. Adams, The Knot Book: An Elementary Introduction to the Mathematical Theory of Knots (Freeman, New York, 1994).

[5] S. A. Wasserman and N. R. Cozzarelli, Science 232, 951 (1986).

[6] A. Krasnow, A. Stasiak, S. J. Spengler, F. Dean, T. Koller, and N. Cozzarelli, Nature (London) 304, 559 (1983).

[7] P. Stazcek and N. P. Higgins, Mol. Microbiol. 29, 1435 (1998).

[8] R. W. Deibler, S. Rahmati, and E. L. Zechiedrich, Genes Dev. 15, 748 (2001).
[9] J. Yan, M. O. Magnasco, and J. F. Marko, Nature (London) 401, 932 (1999).

[10] W. R. Taylor, Nature (London) 406, 916 (2000); M. T. Alam, T. Yamada, U. Carlsson, and A. Ikai, FEBS Lett. 519, 35 (2002); P. Virnau, L. A. Mirny, and M. Kardar, PLOS Comput. Biol. 2, e122 (2006).

[11] P. G. De Gennes, Scaling Concepts in Polymer Physics (Cornell University Press, Ithaca, NY, 1979).

[12] E. J. Janse van Rensburg and S. G. Whittington, J. Phys. A 24, 3935 (1991).

[13] E. Orlandini, M. C. Tesi, E. J. Janse van Rensburg, and S. G. Whittington, J. Phys. A 31, 5953 (1998); O. Farago, Y. Kantor, and M. Kardar, Europhys. Lett. 60, 53 (2002); E. Orlandini, A. L. Stella, and C. Vanderzande, J. Stat. Phys. 115, 681 (2004); P. Virnau, Y. Kantor, and M. Kardar, J. Am. Chem. Soc. 127, 15102 (2005); B. Marcone, E. Orlandini, A. Stella, and F. Zonta, J. Phys. A 38, L15 (2005).

[14] A. Y. Grosberg, Phys. Rev. Lett. 85, 3858 (2000).

[15] R. Metzler, A. Hanke, P. G. Dommersnes, Y. Kantor, and M. Kardar, Phys. Rev. Lett. 88, 188101 (2002).

[16] V. Katritch, W. K. Olson, A. Vologodskii, J. Dubochet, and A. Stasiak, Phys. Rev. E 61, 5545 (2000).

[17] L. F. Liu, L. Perkocha, R. Calendar, and J. C. Wang, Proc. Natl. Acad. Sci. U.S.A. 78, 5498 (1981).

[18] F. B. Dean, A. Stasiak, T. Koller, and N. R. Cozzarelli, J. Biol. Chem. 260, 4975 (1985).

[19] H. Yamaguchi, K. Kubota, and A. Harada, Chem. Lett. 4, 384 (2000).

[20] F. Valle, M. Favre, P. De Los Rios, A. Rosa, and G. Dietler, Phys. Rev. Lett. 95, 158105 (2005).

[21] K. Falconer, Fractal Geometry (John Wiley, Chichester, U.K., 1990).

[22] M. Isaken, B. Julien, R. Calendar, and B. H. Lindgvist, in DNA Topoisomerase Protocols, DNA Topology, and Enzymes, edited by M.A. Bjornsti and N. Osheroff (Humana, Totowa, NJ, 1999), Vol. 94, pp. 69-74.

[23] J. Arsuaga, M. Vazquez, S. Tigueros, D. W. Sumners, and J. Roca, Proc. Natl. Acad. Sci. U.S.A. 99, 5373 (2002).

[24] A. V. Vologodskii, N. J. Crisona, B. Laurie, P. Pieranski, V. Katritch, J. Dubochet, and A. Stasiak, J. Mol. Biol. 278, 1 (1998).

[25] J. Adamcik et al. (to be published).

[26] Y. L. Lyubchenko, A. A. Gall, L. S. Shlyakhtenko, R. E. Harrington, B. L. Jacobs, P. I. Oden, and S. M. Lindsay, J. Biomol. Struct. Dyn. 10, 589 (1992).

[27] A. Dobay, J. Dubochet, K. Millett, P.-E. Sottas, and A. Stasiak, Proc. Natl. Acad. Sci. U.S.A. 100, 5611 (2003).

[28] I. Ippolito, D. Bideau, and A. Hansen, Phys. Rev. E 57, 3656 (1998); I. I. Smailer, J. Machta, and S. Redner, Phys. Rev. E 47, 262 (1993); L. A. Braunstein, S. V. Buldyrev, S. Havlin, and H.E. Stanley, Phys. Rev. E 65, 056128 (2002).

[29] J. Bednar, P. Furrer, V. Katritch, A.Z. Stasiak, J. Dubochet, and A. Stasiak, J. Mol. Biol. 254, 579 (1995).

[30] M. Joanicot and B. Revet, Biopolymers 26, 315 (1987).

[31] C. Rivetti, M. Guthold, and C. Bustamante, J. Mol. Biol. 264, 919 (1996).

[32] A. Hanke, R. Metzler, P. G. Dommersnes, Y. Kantor, and M. Kardar, Eur. Phys. J. E 12, 347 (2003). 